\title{
Man-Portable Networked Sensor System
}

\author{
W. D. Bryan, H. G. Nguyen, and D. W. Gage \\ Space and Naval Warfare Systems Center, San Diego \\ San Diego, California 92152 USA
}

\begin{abstract}
The Man-Portable Networked Sensor System (MPNSS), with its baseline sensor suite of a pan/tilt unit with video and FLIR cameras and laser rangefinder, functions in a distributed network of remote sensing packages and control stations designed to provide a rapidly deployable, extended-range surveillance capability for a wide variety of security operations and other tactical missions.

While first developed as a man-portable prototype, these sensor packages can also be deployed on UGVs and UAVs, and a copy of this package been demonstrated flying on the Sikorsky Cypher VTOL UAV in counterdrug and MOUT scenarios.

The system makes maximum use of COTS components for sensing, processing, and communications, and of both established and emerging standard communications networking protocols and system integration techniques. This paper will discuss the technical issues involved in: (1) system integration using COTS components and emerging bus standards, (2) flexible networking for a scalable system, and (3) the human interface designed to maximize information presentation to the warfighter in battle situations.
\end{abstract}

Key words: security sensor, integration, human interface, network

\section{INTRODUCTION}

In today's environment of Operations Other Than War, military personnel are increasingly called upon to enter urban or similarly complex hostile environments and encounter life-threatening situations. The typical high risk conditions the warfighters are exposed to include clearing buildings, neutralizing snipers, and rescuing hostages while avoiding ambush.

The Man-Portable Network Sensor System (MPNSS) has been developed to reduce risk to military personnel by improving their situational awareness and increasing squad efficiency in executing these tactical mission tasks [1]. It consists of a network of portable sensors that can be placed by the soldiers at strategic locations to provide continuous surveillance of critical areas (Figure 1). Still images and continuous video (in both visible light and infrared), motion and acoustic alerts, and range to targets are available to remote control/display stations linked to the radio-frequency (RF) network. Control/display stations can be portable laptop computers, handheld terminal units, soldier-wearable computers or vehicle-mounted computers.

MPNSS has roots in the Air Mobile Ground Security and Surveillance System (AMGSSS) project [2,3], started in fiscal year 1992. The AMGSSS project's goal was to provide a rapidly deployable, extended-range surveillance capability for force protection and tactical security. It consisted of three vertical-takeoff-and-landing (VTOL) air-mobile remote sensing platforms (Figure 2) and a control station. The VTOL air-mobile platform's function is to carry its sensor payload to remote ground locations, up to $10 \mathrm{~km}$ from the base station, for extended surveillance missions. These ground locations can be hill tops, roof tops, or any place with clear view of critical areas. This program was supported by the US Army Product ManagerPhysical Security Equipment (PM-PSE) and the Office of the Undersecretary of Defense (Acquisition, Tactical Systems/Land Systems).

In 1996 AMGSSS acquired additional support from the US Army Military Police School and the US Army Infantry Center Dismounted Battlespace Battle Laboratory, and changed its name to the Multipurpose Security and Surveillance 


\section{Report Documentation Page}

Form Approved OMB No. 0704-0188

Public reporting burden for the collection of information is estimated to average 1 hour per response, including the time for reviewing instructions, searching existing data sources, gathering and maintaining the data needed, and completing and reviewing the collection of information. Send comments regarding this burden estimate or any other aspect of this collection of information,

including suggestions for reducing this burden, to Washington Headquarters Services, Directorate for Information Operations and Reports, 1215 Jefferson Davis Highway, Suite 1204, Arlington

VA 22202-4302. Respondents should be aware that notwithstanding any other provision of law, no person shall be subject to a penalty for failing to comply with a collection of information if it

does not display a currently valid OMB control number.

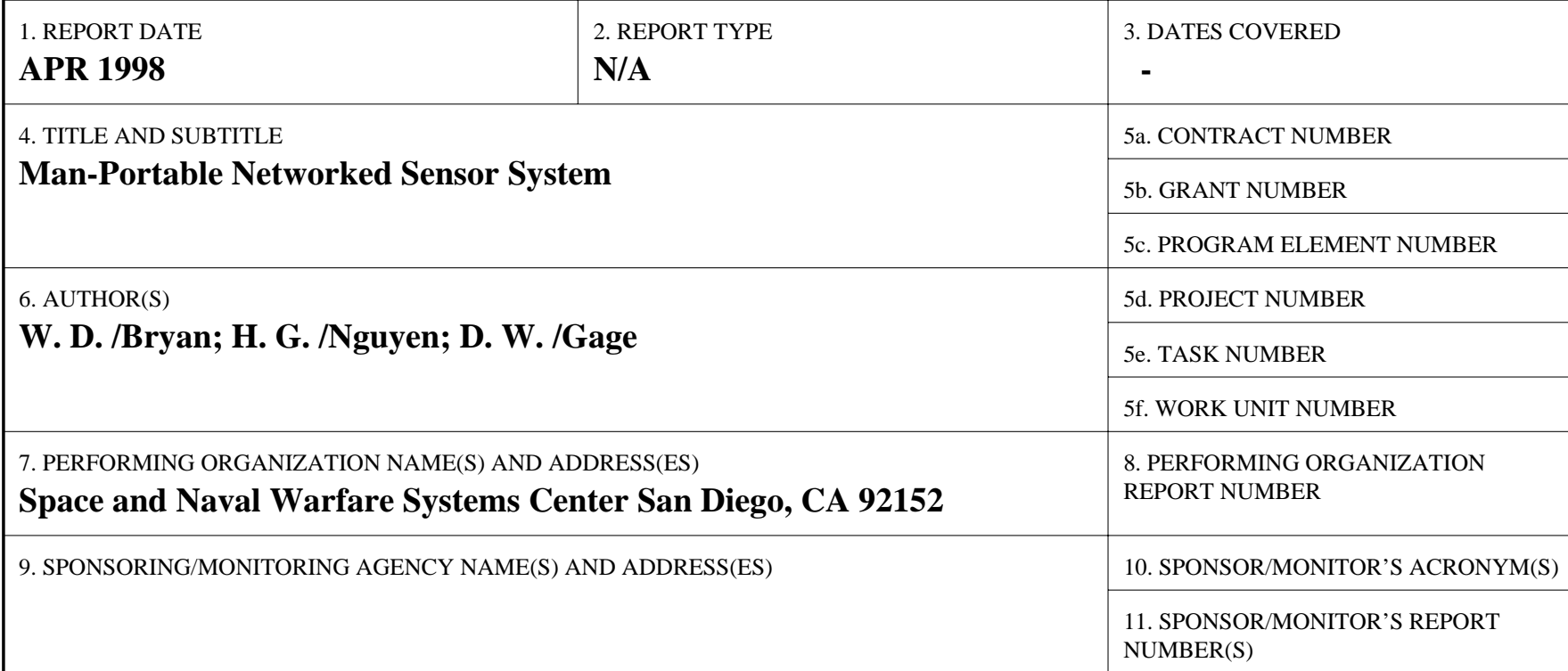

12. DISTRIBUTION/AVAILABILITY STATEMENT

Approved for public release, distribution unlimited

13. SUPPLEMENTARY NOTES

14. ABSTRACT

15. SUBJECT TERMS

16. SECURITY CLASSIFICATION OF:

\begin{tabular}{c|c|c|}
$\begin{array}{c}\text { a. REPORT } \\
\text { unclassified }\end{array}$ & $\begin{array}{c}\text { b. ABSTRACT } \\
\text { unclassified }\end{array}$ & $\begin{array}{c}\text { c. THIS PAGE } \\
\text { unclassified }\end{array}$ \\
\hline
\end{tabular}

17. LIMITATION OF
ABSTRACT
UU

\begin{tabular}{c|l}
$\begin{array}{c}\text { 18. NUMBER } \\
\text { OF PAGES } \\
\mathbf{1 0}\end{array}$ & 19a. NAME OF \\
& \\
&
\end{tabular}




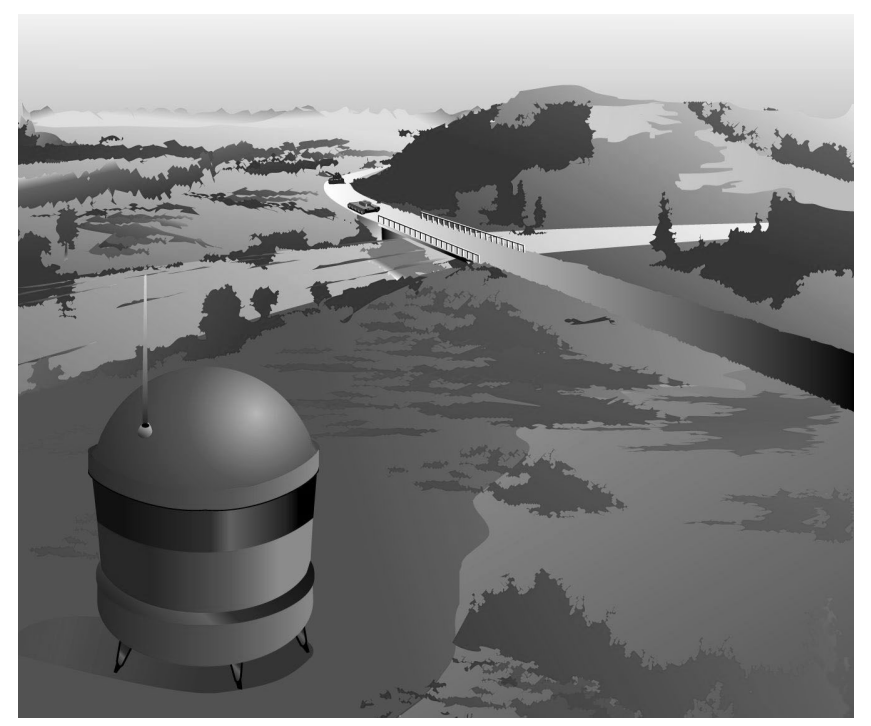

Figure 1. Artist's concept of the MPNSS ruggedized sensor package.

Mission Platform (MSSMP) [4,5]. The system's role expanded to include new mission areas such as: support to counterdrug and border patrol operations, detection and assessment of barriers (i.e., mine fields, tank traps), remote assessment of suspected contaminated areas (i.e., chemical, biological, and nuclear), and the use of the air-mobile platform as signal/communications relays and to resupply small quantities of critical items.

In 1997, two MSSMP-related Technology Base efforts supported by the Defense Special Weapons Agency were initiated. The first project deals with issues relevant to tactical security sensor internetting and integration [6,7]. One focus is on the identification or definition of Internet Protocol (IP)-based protocols tuned to the requirement for message-based traffic over a heterogeneous RF network whose throughput and delay characteristics are likely to vary in both space and time. Such a protocol must, unlike the Transmission Control Protocol (TCP), provide real-time communications status information in order to allow both the operator and the remote sensor package to adapt their behavior to changing communications capacities in real time. A second focus is on identifying commercial-off-the-shelf (COTS) distributed control techniques that can be used to expedite the effective integration of COTS sensor and other subsystems that provide only slow, asynchronous, character-based RS-232 control interfaces.

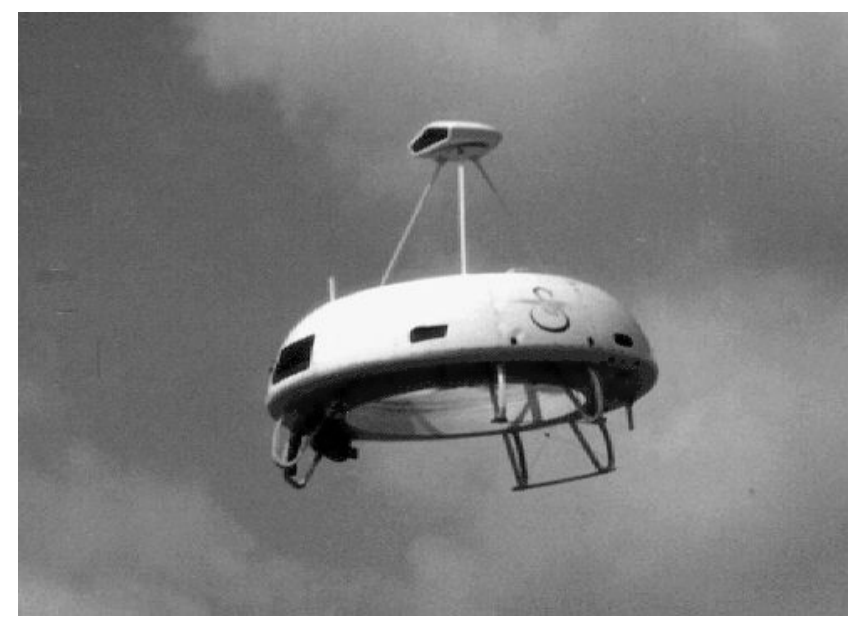

Figure 2. The AMGSSS/MSSMP air-mobile platform and integrated sensor pod. 
The second project is investigating more advanced human interface techniques for presenting tactical sensor information intuitively and intelligently to the warfighter, preventing information overload during stressful conditions. Topics that are being studied include:

- Alternative input mechanisms (e.g., voice, gesture)

- Alternative displays and cueing mechanisms (e.g., see-through displays, audio cueing)

- More efficient, direct graphic presentation

The findings of this second project, together with experience gained with the portable prototype sensor package during field testing of the AMGSSS/MSSMP system at Military-Operations-Urban-Terrain (MOUT) facilities [5], led us to realize the importance of a man-portable networked sensor system. Without the cost of the air-mobile platforms, a network of manportable sensor units can be inexpensively produced that will make significant contributions to urban terrain operations. Funding has been obtained to initiate an effort to produce a ruggedized sensor package and incorporate improvements which came out of the AMGSSS/MSSMP system tests.

MPNSS inherits from its predecessors not only a baseline prototype sensor package, but also the following important features:

- The use commercial-off-the-shelf (COTS) sensors and standards-based hardware and software

- A flexible Internet Protocol (IP)-based networked communications architecture

The incorporation of these features enabled the rapid prototyping of a flexible system that can be readily expanded or modified [3].

\section{SENSOR SYSTEM}

The MPNSS system consists of a scalable suite of smart sensors configured into man-portable packages. Multiple sensor packages are distributed throughout a surveillance area and are linked together by a wireless network communications architecture. Sensor/control data from/to these sensor nodes is available to users throughout this network.

The majority of sensory data processing is performed at the remote sensor node. This reduces both the bandwidth and the power consumption required to transmit the information. Decisions are made by the remote computers so that only useful data is transmitted. Communications only occur when there is information to communicate.

The system architecture is flexible enough to allow easy integration of future sensors and the replacement of current sensors with more advanced models. This architecture provides for scalability: a given sensor or network of sensors can be easily configured to satisfy the mission functional requirements. A sensor package can consist of a single sensor such as a helmet-mounted video camera, an unattended ground sensor, or a suite of integrated sensors. The baseline sensor suite (Figure 3a) provides the following functionality: video/images for visual target classification, image enhancement, motion detection and acoustic alerts, acoustic classification, sensor/image processing for bandwidth reduction, and GPS/heading/range data processing for absolute target positioning.

\subsection{MPNSS Baseline Sensors}

The baseline MPNSS sensor suite (Figure 3a) includes [5]:

- Visible light video camera

- Infrared video camera (FLIR)

- Laser rangefinder

- Global Positioning System (GPS) receiver and heading sensor

- Interface for an acoustic sensor

- Interface for legacy sensor gateway 
Sensor selections were guided by the criteria of minimum power, size and weight, as well as adequacy of performance, low cost and off-the-shelf availability at integration time.

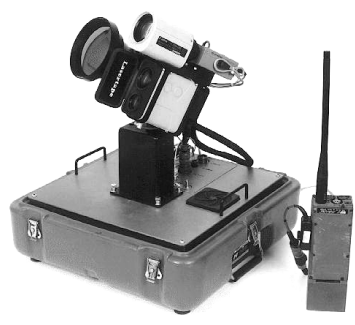

(a)

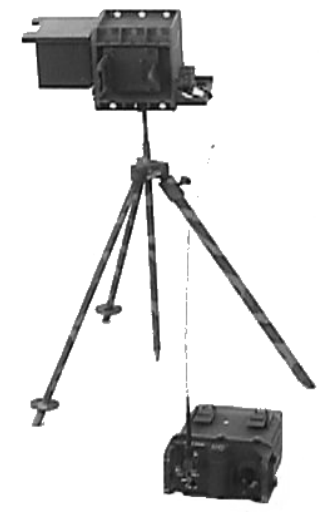

(b)

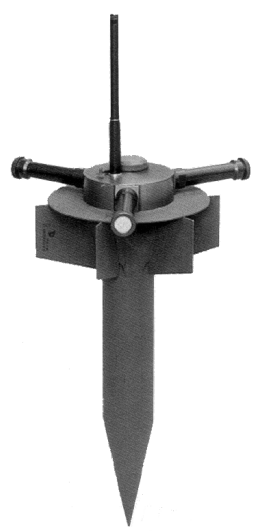

(c)

Figure 3. Tested MPNSS sensors: (a) Integrated sensor package, (b) Tactical Remote Sensor System (TRSS) ground sensor,

(c) Northrop Grumman's Unattended Ground Sensor. In addition, the MPNSS sensors can also be mounted on VTOL unmanned air vehicles (UAVs) and unmanned ground vehicles (UGVs), as shown in Figure 4.

\section{Visible-Light Video Camera:}

A Cohu camera with a Canon 10:1 zoom lens and a $2 \mathrm{X}$ range extender was selected. Design specifications called for monochrome images with sufficient resolution to allow classification of vehicles at $2.5 \mathrm{~km}$ and personnel at $1 \mathrm{~km}$. This setup allows us to meet the Johnson Criteria [8] for resolution, as well as the sensitivity, dynamic range and signal-to-noise ratio necessary for reliable target detection and classification at those distances.

\section{Infrared Video Camera:}

The thermal imager is required to meet the same requirements as the visible-light imaging system but in total darkness. An Inframetrics InfraCam was selected, with a 100mm lens. This imager uses a platinum silicide focal plane array for high pixel uniformity and a proprietary Stirling-cycle dewar cooler to combine light weight with low power and reasonably high image quality.

\section{Laser Rangefinder:}

To determine target range, a laser rangefinder was included in the sensor suite. Minimum MPNSS requirement for a laser rangefinder is the ability to reliably determine the range of typical military targets at up to 2,500 meters with 10 meters accuracy. The unit must also be class-1 eyesafe and remotely controllable.

Nine laser rangefinders were found which met the requirements to varying degrees. The Melios, in wide use in the US Army, would probably be the best choice for a fieldable system. However, to save money and weight, we compromised and used a Riegl Lasertape for the prototype system. The Lasertape works well to about 800 meters, sufficient for many applications.

\section{GPS/Heading:}

Target position data is determined using a combination of sensor data including GPS, heading, and range. Rockwell's Zodiac sensor, a 12 channel GPS receiver, provides sensor package position data. This sensor comes with an additional port for differential correction updates for improved position precision. Differential updates are transmitted using the existing RF 
communications network. A heading sensor from Precision Navigation provides target bearing data corrected for sensor tilt and magnetic distortions. The tilt sensor is also used to monitor for disturbance of deployed sensor package. Onboard processing of this data along with range data provides accurate position information of surveillance targets.

\section{Acoustic Detector:}

Because of the immaturity and rapid evolution of product development of acoustic detectors, we decided to provide an external data connection for an optional acoustic sensor instead of physically integrating one into the sensor package. A prototype air-deployed acoustic sensor made by Northrop-Grumman (Figure 3c) was connected to the MSSMP Mission Payload Prototype and tested in the field in late 1995. The acoustic package is a small ring of three microphones with custom processing hardware and a serial interface. Output indicates target azimuth angle, type (ground vehicle, jet, helicopter, etc.), and detection/ classification confidence. MPNSS software provides programmable filters that allow the user to discard specified types of sound from specified azimuthal areas.

\section{Legacy Sensor System Gateway:}

Legacy sensor systems fielded in the services, such as the Tactical Remote Sensor System (TRSS), Improved Remotely Monitored Battlefield Sensor System (IREMBASS), and Tactical Automated Security System (TASS), employ a standard communications protocol (SEIWG-05) for transmitting sensor data over very-high-frequency (VHF) point-to-point radio links. A small VHF transceiver is embedded in the MPNSS baseline sensor package delivering this sensor data to the payload processor. Software has been written to monitor alerts from these sensor systems and reformat the data into IP packets. This data is multiplexed along with other sensor data and distributed across the network to all users.

A more detailed description of MPNSS sensors can be found in [9].

\subsection{Sensor Processing Architecture}

Three microcomputers reside in the MPNSS baseline sensor package, processing and reducing the raw data collected before transmitting them back to the Control/Display unit. These take the form of low-power PC/104 form-factor processor boards, running the MS-DOS and MS-Windows 95 operating systems. The three payload computers are interconnected using an Ethernet link for inter-processor communications. Each computer has its own Internet address, and theoretically could be thousands of miles apart, connected by any available IP-based infrastructure, wired or wireless. This architecture has allowed parallel development of the subsystems at different sites, easy debugging by substitution of any processor by an equivalent desktop computer, and has produced a flexible system that can be readily expanded or modified [3].

The Payload Processor handles the communication with the Control/Display, interpreting and executing high level Control/Display commands by generating and sending sequences of simple low-level commands to the various sensor subsystems via RS-232 and other interfaces. It also coordinates the flow of information between all payload computers; monitors, filters, and consolidates alerts received from the Image Processor, acoustic detector, and legacy sensor gateway; and periodically sends status updates for all sensors to the Control/Display unit.

The Image Processor compresses images before sending them back to the Control/Display, using JPEG compression, and performs scene video motion detection when commanded. Image enhancement techniques (contrast enhancement, histogram equalization, etc.), when selected by the operator, are also performed by the Image Processor on the captured image before compression and transmission.

The Video Processor is dedicated to real-time video compressing and transmission. A commercial Windows-95 CUSeeMe software package is used to perform this function. This package features multicast capability which provides IPbased voice and video to multiple users operating within the surveillance network. Currently, the video and voice functions are being upgraded to an embedded hardware codec card which uses H.320/H.323 compression algorithms for improved performance.

The baseline MPNSS package has taken advantage of recent advances in COTS processor performance and component integration driven by the PC notebook computer market. This has resulted in an increase in sensor performance while reducing sensor package power consumption, size, and weight. 


\section{COMMUNICATIONS NETWORK}

The MPNSS communications architecture consists of an all-digital fully-connected adaptive network (Figure 4) providing integrated video, voice, and data services. The fundamental component of this architecture is its basis on the IP suite. This enables plug-and-play compatibility with existing wired and wireless commercial/government off-the-shelf (COTS/GOTS) communications hardware, including evolving Army tactical communications architectures and systems, such as Tactical Internet (TI), Near-Term Digital Radio (NTDR), Joint Tactical Radio System (JTRS), and Warfighter Information Network (WIN). Operational tests this year will demonstrate a mix of COTS/GOTS wireless systems including the Army's NTDR tactical radio, SEIWG-05 radios, COTS 802.11 wireless local area network (WLAN) radios, and Cellular Digital Packet Data (CDPD) modems. Features of these tests will include: (1) on-the-move connectivity from sensor to soldier, soldier to soldier, and sensor to tactical operation centers; (2) integration of legacy sensor systems (TRSS, IREMBASS) through network gateway hardware; and (3) beyond-line-of-sight (BLOS) sensor access/control.

The communications architecture was initially developed using SINCGARS and PRC-139 tactical radios [5,10]. The SINCGARS radio is the Army's projected least common denominator Combat Net Radio. Magnavox/SAIC PCMCIA Tactical Communication Interface Modules (TCIMs), with software modified by us, provided the physical layer for the IPbased network connectivity in accordance with MIL-188-220A over SINCGARS radios. Operational field tests were conducted in 1995, and beyond-line-of-sight network connectivity was established with this tactical IP data link. However, effective data throughput was low ( $<500 \mathrm{bps}$ ), and COTS WLAN technology was subsequently substituted to improve system performance until military communications technology catches up.

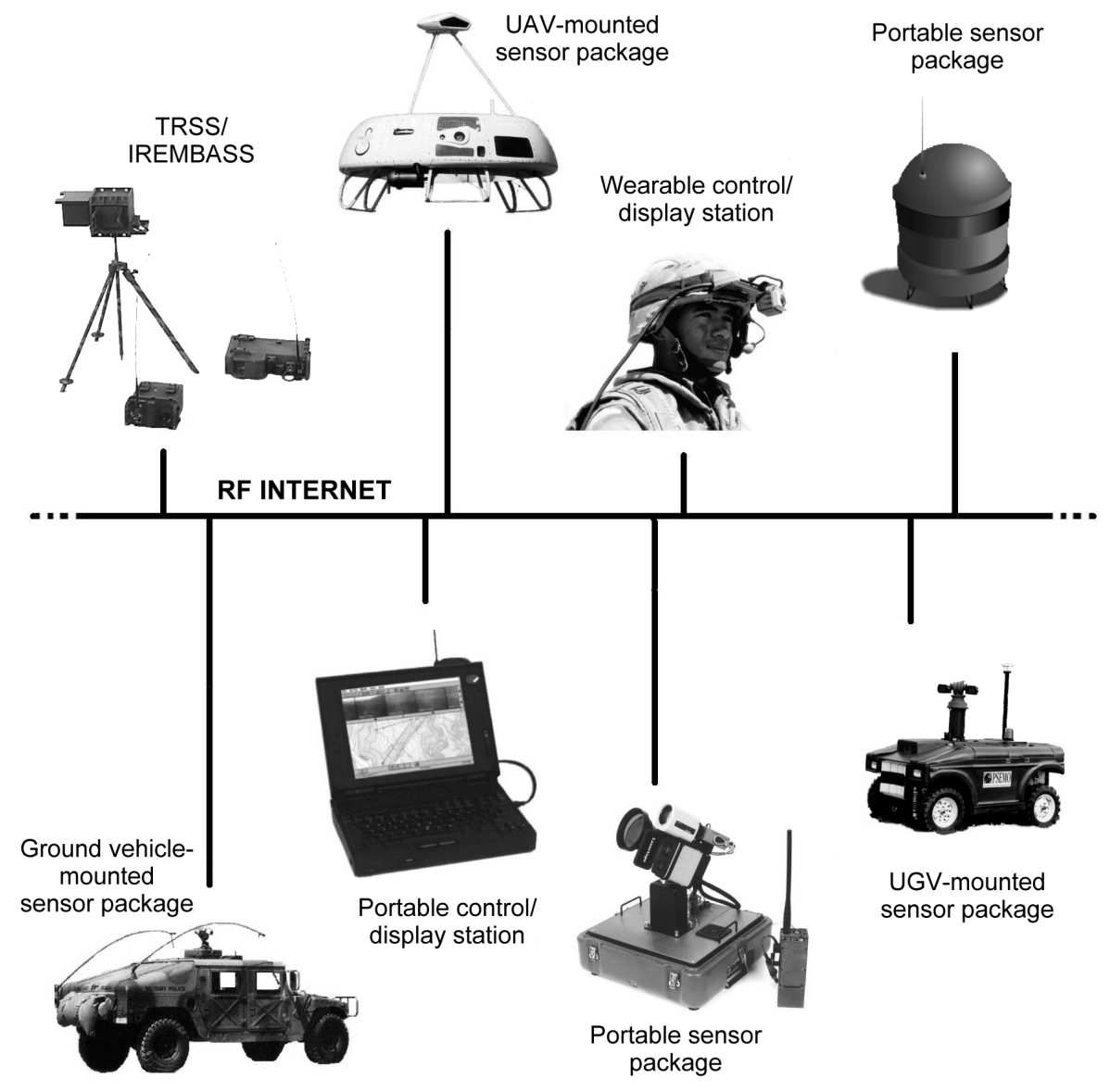

Figure 4. The MPNSS scalable system architecture 
The current MPNSS communications architecture provides the following features:

\section{Beyond-line-of-sight communications:}

BLOS communications is provided through a combination of multiple line-of-sight (LOS) network communication segments. The IP-based radios self-organize into a tree structured network topology. Each radio maintains a dynamic configuration table of radio node connections within the communications network. IP devices connected to each radio are also listed in the configuration table. This table is used to automatically route data between source and destination nodes. If source and destination nodes are BLOS, the data is routed (hopped) across multiple LOS network segments until it reaches its destination node. If a radio node goes down, radio configuration tables are updated and the data packets passing through the down radio node are rerouted automatically. The Spanning Tree Protocol (IEEE 802.1d) is used to prevent data routing loops from developing and reducing available network bandwidth.

\section{Scalability and Mobility:}

Radio nodes (associated with sensors, warfighters, and control stations) are automatically added to or deleted from the network configuration table as they enter or leave the network. A mobile node can "roam" through the network and maintain connectivity. The mobile node is automatically connected to another node (static or mobile) in the vicinity of maneuver. The network topology is updated and data traffic is rerouted to arrive at the new mobile node position without the need of operator intervention.

\section{Integrated voice, video, and data services:}

Data packets containing video, voice, and sensor data are multiplexed onto the same RF channel. These packets can be transmitted point-to-point (unicast), point-to-multipoint (broadcast), or multipoint-to-multipoint (multicast). Different types of digital data (voice, video, sensor, and control) are converted to IP data packets by application software. Standard IP network software then performs the function of data multiplexing/demultiplexing automatically. This simple scheme of multiplexing services eliminates the requirement for separate communications systems that have traditionally been needed to provide for each of voice communications, video transmission, and sensor command and control. However, latency-critical services like voice can experience degraded performance on congested networks.

\section{IP gateway for military legacy sensor systems:}

The US Department of Defense (DoD) currently has several sensor systems in its inventory including the Marine Corps' TRSS, the Army's IREMBASS, and the Air Force's TASS systems. All these systems use a standard radio communication scheme based on SEIWG-05. Each of these systems represents a stovepipe architecture whereby sensor data is available only to a given sensor display station. An IP-based gateway is being developed to enable DoD legacy sensor data to be accessed by multiple users over a widely distributed network. This wireless gateway reformats the point-to-point legacy sensor data into IP packets which can be distributed horizontally across the MPNSS communications network and vertically to higher echelon commands using TI and WIN.

\section{Flexible mix of existing communications channels:}

The IP-based network architecture takes advantage of the vast communication infrastructures, both wired and wireless, that have been developed to pass IP data traffic. MPNSS has been seamlessly demonstrated using a heterogeneous set of communications hardware including tactical radios, COTS WLAN radios, the Internet, Cellular, and Plain Old Telephone System (POTS). Communications tests this year will consists of a mix of COTS/GOTS wireless systems including the Army's NTDR tactical radio, SEIWG-05 radios, 802.11 WLAN radios, and CDPD modems. This flexible mix of communication links and the existing Internet infrastructure enables an extremely wide and flexible network, allowing remote monitoring or control of field sensors by command echelons over vast distances.

Current network communication issues being investigated [6,7] are robust transport protocol development for mobile wireless networks, efficient mechanisms for network data distribution, and intelligent arbitration techniques for control of a sensor by multiple users in a network. 


\section{HUMAN INTERFACE}

The user interface for the MPNSS is a Microsoft Windows-based software program with standard windows, menus, buttons, and dialog boxes. Using a keyboard and mouse, the operator can display remote sensor data, command remote sensors to perform elementary functions--including taking target snapshots (at specified zoom, focus, gain, polarity, azimuth and elevation, etc.), enhancing target images prior to transmission, measuring target range/position, initiating live compressed video of target--or program complex sequences of commands such as composing image panoramas, acoustic filters, and motion detection at various critical scene points. An in-depth discussion of how these commands are executed can be found in [9]. Figure 5 shows examples of two Control/Display screens that provide situational awareness to the operator (details of the Control/Display features were presented in [3]).

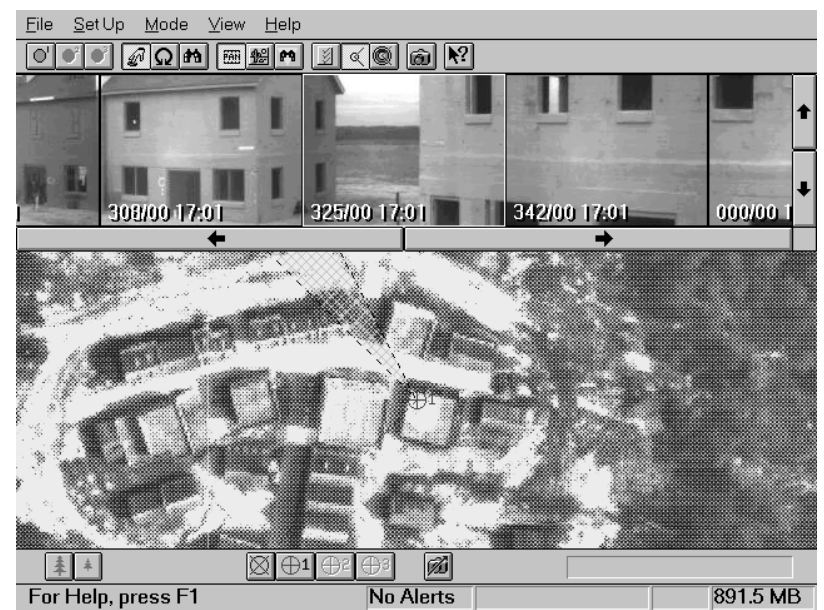

(a)

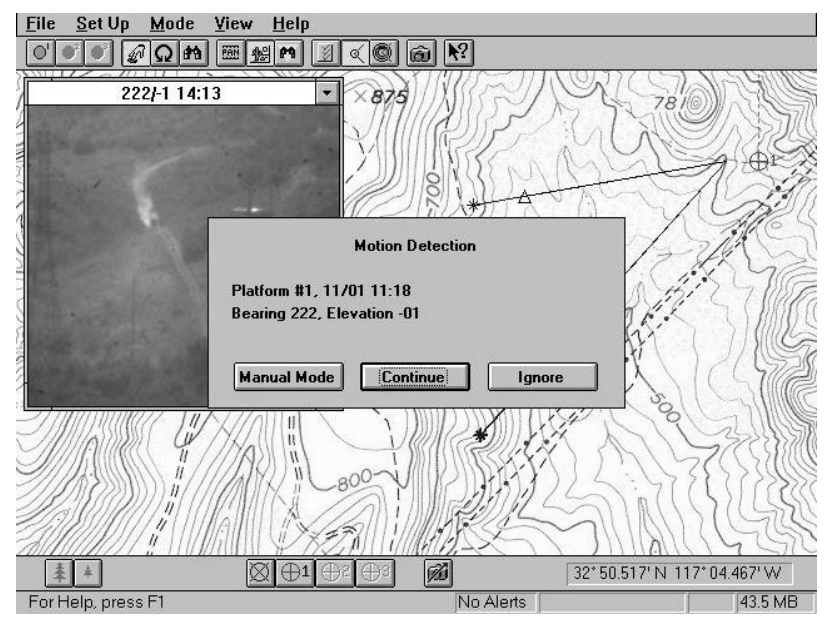

(b)

Figure 5. Two sample Control/Display screens: (a) Ft. Benning panorama and corresponding aerial photo, (b) Motion detection alert, target image and location on map.

While the Control/Display program was first developed for laptop computers, we have also successfully demonstrated it on a pen computer, a Xybernaut soldier-wearable computer, and the Litton Handheld Terminal Unit (one of the prototype Force XXI Dismounted Soldier System Units) [5] (see Figure 6). The wearable computer tested with the MPNSS system includes a small computer (with integrated trackball) and battery pack that can be worn around the waist, and a small headmounted display (over one eye) with integrated camera, microphone and earphone. This configuration added several demonstrated capabilities to the system:

- The warfighter can access, monitor, and control remote sensor data while on-the-move.

- Voice control and feedback of sensor system functions provides hands free operation.

- Heads-up display provides viewing of remote sensor data within the warfighter's field of view.

- Video from the head-mounted camera enables the warfighter to function as a sensor within the network.

- Integrated voice communications within the communications network architecture.

Current research efforts are underway to develop more intuitive methods for presenting/controlling tactical sensor data to/by the warfighter that will improve his situational awareness without adding additional stress while performing tactical mission scenarios. This research specifically addresses new metaphors of input/output for the human-computer interface. Input metaphors being investigated include voice, gesture, and touch. Output metaphors include augmented display technology, and retinal, symbolic, audio, and tactile feedback. Wearable and vehicle computer hardware technologies are being investigated for both mounted and dismounted tactical scenarios. 


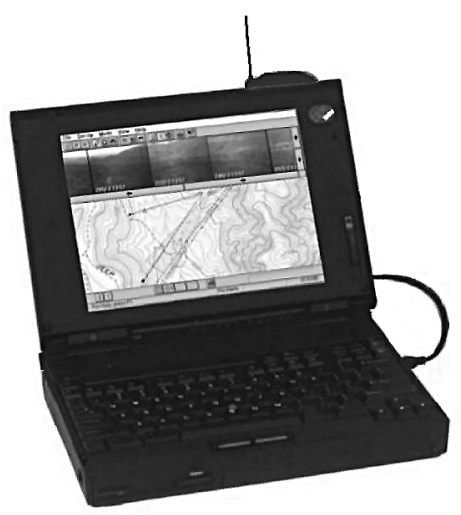

(a)

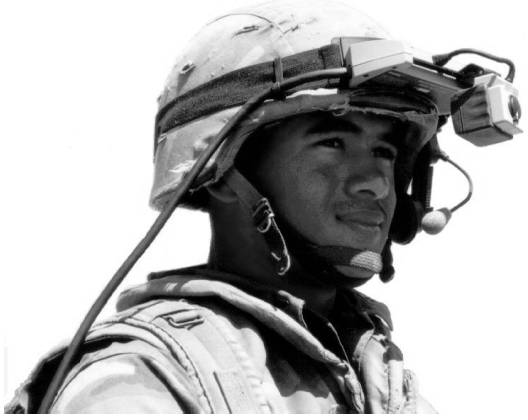

(b)

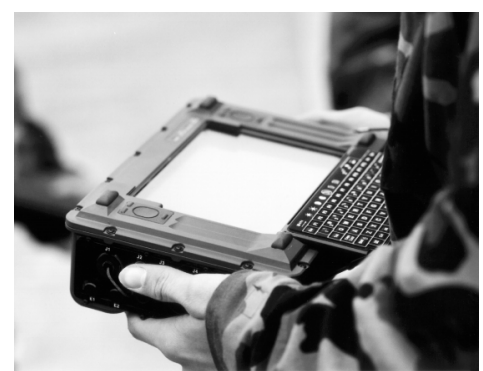

(c)

Figure 6. Some tested Control/Display configurations: (a) laptop computer, (b) wearable computer, (c) Dismounted Soldier System Unit.

\section{CONCLUSION}

The Man-Portable Networked Sensor System is a flexible, readily extendable network of portable sensors designed to provide improved situation awareness, security, and surveillance capabilities to the warfighter. Sensors may include any combination of the portable baseline integrated sensor packages (with COTS video, infrared, and laser-rangefinding sensors), to new prototype or DoD legacy acoustic ground sensors. The IP based network takes advantage of the myriad of communications channels that have been developed for the Internet, providing a flexible network that allows command, control and monitoring of field sensors by warfighters and command echelons over vast distances.

In January 1997, the prototype MPNSS baseline sensor package was demonstrated (in the same wireless network with its UAV-mounted counterpart) at the US Army's MOUT facility at Ft. Benning, Georgia [5]. The system demonstrated reconnaissance support for advancing troops and security surveillance in urban terrain. In August 1997, the MPNSS portable baseline sensor package was operated in the stand-alone configuration during MOUT exercises at Camp Pendleton Marine Corps Base in California.

Also in 1997, at the request of Naval Air Station Keflavik, Iceland, we developed test plans to demonstrate the long-term, severe-weather use of MPNSS for augmenting existing base security measures. MPNSS sensors would remotely guard aircraft bunkers, flightline, and base perimeter, reducing manning requirements that are costly in the often hostile Icelandic weather. The wireless MPNSS network provides the flexibility needed to easily extend the base security perimeter outward during periods of heightened threat conditions.

Together with on-going technical-base efforts, funded by the Defense Special Weapons Agency, to develop advanced human interface and networking technologies, work has begun on ruggedizing the MPNSS integrated sensor package in anticipation of the tests in Iceland.

\section{REFERENCES}

1. "Man-Portable Networked Sensor System," WWW URL: http://marlin.spawar.navy.mil/D37/D371/mpnss/mpnss.html.

2. D. W. Murphy and J. P. Bott, "On the Lookout: The Air Mobile Ground Security and Surveillance System (AMGSSS) Has Arrived,” Unmanned Systems, Vol. 13, No. 4, Fall 1995.

3. H. G. Nguyen, W. C. Marsh, and W. D. Bryan, "Virtual Systems: Aspects of the Air-Mobile Ground Security and Surveillance System Prototype," Unmanned Systems, Vol. 14, No. 1, Winter 1996. 
4. "Multipurpose Security and Surveillance Mission Platform," http://www.spawar.navy.mil/robots/air/amgsss/mssmp.html.

5. D. W. Murphy, J. P. Bott, W. D. Bryan, J. L. Coleman, D. W. Gage, H. G. Nguyen, and M. P. Cheatham, "MSSMP: No Place to Hide," AUVSI '97 Proceedings, pp. 281-290, Baltimore, MD, 3-6 June, 1997.

6. D. W. Gage, "Network Protocols for Mobile Robot Systems," SPIE Proc. 3210: Mobile Robots XII, Pittsburgh, PA, 1417 October, 1997.

7. D. W. Gage, W. D. Bryan, and H. G. Nguyen, "Internetting Tactical Security Sensor Systems," SPIE Vol. 3393: Digitization of the Battlespace, Orlando, FL, 15-17 April 1998.

8. J. Johnson, "Analysis of Image Forming Systems," Proc. Image Intensifier Symposium, US Army Engineer Research and Development Laboratories, Fort Belvoir, VA, 6-7 Oct. 1958 (AD220160).

9. D. W. Murphy et al., "Air-Mobile Ground Security and Surveillance System (AMGSSS) Project Summary Report," Technical Document 2914, NCCOSC RDT\&E Div., San Diego, CA, September 1996 (AD-A317618).

10. B. Martin and D. Bryan, "Low-Cost Miniature Interface and Control Systems for Smart Sensors, Tactical Radios, and Computer Networks," IEEE Military Communications Conference (MILCOM 95), San Diego, CA, Nov. 6-8, 1995. 